\title{
Hospital and Community Pharmacists' Views of and Perspectives on the Establishment of an Intraprofessional Collaboration in the Transition of Care for Newly Discharged Patients
}

Laura V.J. Lech, MSc Pharm ${ }^{1}$; Gitte R. Husted, MScN, PhD²; Anna Birna Almarsdottír, MSc Pharm, PhD';

Trine R.H. Andersen, MSc Pharm, PhD ; Charlotte Rossing, MSc Pharm, PhD ${ }^{2}$; Lotte S. Nørgaard, MSc Pharm, PhD 1

${ }^{1}$ The Social and Clinical Pharmacy Research Group, Department of Pharmacy, Faculty of Health and Medical Sciences, University of

Copenhagen; ${ }^{2}$ Pharmakon, The Danish College of Pharmacy Practice; ${ }^{3}$ Region Zealand Hospital Pharmacy

\section{ABSTRACT}

Background Hospital and community pharmacists are increasingly involved in patients' medication interventions related to the transition of care from the hospital to a patient's home. These interventions may enable pharmacists to collaborate across healthcare sector boundaries. However, little is known about pharmacists' views on intraprofessional collaboration across healthcare sectors and what affects the establishment of such collaboration.

Objectives The aim of this study was to understand the views and perspectives of hospital pharmacists (HPs) and community pharmacists (CPs) on establishing an intraprofessional collaboration in relation to newly discharging patients.

Methods Joint focus group interviews with HPs and CPs in the Zealand region of Denmark were conducted. The HPs were employed at the only hospital pharmacy in the region (Region Zealand Hospital Pharmacy). Five HPs and six CPs participated in two focus groups. The focus groups were analyzed using theoretical thematic analysis.

Results Three themes and seven subthemes were identified. The first theme, "Context", was divided into two subthemes: "Prioritization of new tasks in the intraprofessional collaboration" and "The lack of insight into the patient's hospital stay". The second theme, "The hospital physicians and GPs as the focal points for the HPs' and CPs' work", was divided into three subthemes: "The limitation of the CPs and HPs based on current roles and organizations", "Lack of regular access to the physician gives the patients more responsibility" and "Lack of support from the GPs for the work conducted by HPs and CPs". The last theme, "Individuals", had two subthemes: "The motivation for working intraprofessionally" and "CPs' hesitancy towards the new tasks in the intraprofessional collaboration".

Conclusion Both HPs and CPs are highly influenced by their work context in regard to establishing a new intraprofessional collaboration. Limited resources for intraprofessional collaboration should be taken into account. Likewise, the collaboration should fit into daily routines, which may eliminate hesitancy towards new forms of collaboration and tasks shared between HPs and CPs. The physician was identified as an important key professional, since the work tasks of both HPs and CPs depend on the physician, which in turn limits the capability and success of solely intraprofessional collaboration.

Keywords: Hospital pharmacy, hospital discharge, transition of care, intraprofessional collaboration, community pharmacy

\section{INTRODUCTION}

The transition of care is a critical point for patient medication safety, especially when patients are discharged from the hospital to primary care [1-7]. The decision to discharge a patient resides in the hospital, and a majority of studies published have been hospital-based and have sometimes included hospital pharmacists (HPs) [8-12]. The pharmacist's role in optimizing the transition of care has been well studied, and among other things, the studies highlight close collaboration with other professions as a pivotal component in creating safe transitions for patients [13-16]. One way of securing a seamless transition of care is through pharmacist involvement in multidisciplinary care teams collaborating with other professions, in particular hospital physicians and general

Corresponding author: Laura V.J. Lech, MSc Pharm

The Social and Clinical Pharmacy Research Group, Department of Pharmacy, Faculty of Health and

Medical Sciences, University of Copenhagen

Universitetsparken 2, 2100 København K, Denmark

Phone: + 45536507 29; E-mail: laura.lech@sund.ku.dk

Orchid-ID: 0000-0002-9771-9227 practitioners (GPs) [9,17]. The pharmacist's role in multidisciplinary teams includes acting as the medication expert, providing advice, and suggesting interventions for how to secure rational pharmacotherapy and patient safety. This involvement and interprofessional collaboration have yielded significant positive effects on both clinical endpoints, especially hard endpoints such as hospital readmissions [18-20]. Interprofessional collaboration with physicians is slowly becoming well established in some countries in hospitals and GP practices. Gobis et al., however, suggest that more research should be conducted in the field of intraprofessional pharmacist-to-pharmacist collaboration, especially at transition of care points, to avoid a fragmented pharmaceutical approach to patient care [21].

Few studies have described interventions involving intraprofessional collaboration between hospital and community pharmacists. These interventions involve information sharing regarding medication changes and relevant patient information from the HPs to the community pharmacists (CPs), enabling the CPs to gain access to relevant information needed to inform newly discharged patients. The information sharing either resulted in a new intervention conducted for the patients at the community pharmacy or in 
improved counselling of the patients at the community pharmacy $[10,22-26]$.

In a survey conducted by the European Association of Hospital Pharmacists (EAHP) it was concluded that more than half of the CPs had never been contacted by an HP, and over half of both HPs and CPs found it either essential or important to collaborate at especially transition of care points [27]. Thus, there is a demand expressed by both HPs and CPs for more intraprofessional collaboration. However, literature is lacking in relation to understanding HPs' and CPs' views and perspectives on establishing such intraprofessional collaboration at care transitions. Understanding the views and perspectives of HPs and CPs might shed light on existing barriers or facilitators that can affect the feasibility of intraprofessional collaboration in a cross-sector setting.

In Denmark, HPs are involved in various parts of the patient's hospital stay, at admission, during the hospital stay and at discharge, performing a broad range of services, such as medication reviews, medication reconciliation and discharge conversations. This study is a part of a larger study conducted within Region Zealand in Denmark. In Region Zealand, HPs are routinely involved in admission of patients on the acute wards, where they compile medication history and conduct a medication reconciliation and a medication review [28]. In Region Zealand, HPs do not routinely take part in discharging patients nor do they routinely collaborate or send information directly to the CPs at the community pharmacy. Thus, this study explores the views and perspectives of CPs and HPs on a new potential intraprofessional collaboration.

\section{OBJECTIVE(S)}

The aim of this study was to understand the views and perspectives of hospital pharmacists (HPs) and community pharmacists (CPs) on establishing an intraprofessional collaboration concerning newly discharged patients.

\section{ETHICS APPROVAL}

Since participation in the focus groups involved recording of personal data (e.g., age, gender workplace and work experience), permission for the study was obtained from the Danish Data Protection Agency through the Faculty of Health and Medical Sciences, University of Copenhagen, with reference number: 514-0264/18-3000.

\section{METHODS}

An explorative qualitative design comprising focus group interviews was chosen. Focus group interviews are especially suitable for grasping the joint perspectives and reflections of a specific population [29,30]. Additionally, the interaction between participants stimulates discussions in a natural way, a feature specifically useful when exploring the opinions of the two groups of pharmacists (HPs and CPs), who are not collaborating already [29].

\section{Setting}

The study was conducted in Region Zealand in Denmark which covers 38 community pharmacies. The community pharmacies offer free services for patients, for example, medication conversation, which is a consultation service for patients being prescribed medicine for a newly diagnosed chronic disease or for medication non-adherent chronic medication-users [31]. The medication conversation was developed in England as a socalled "New medicine-service" and is described in more detail in the protocol by Boyd. et al. [32].

Region Zealand has one regional hospital pharmacy. The pharmacy provides medication and clinical pharmacy services to the eight hospitals in the region as well as the psychiatric hospital. One of the clinical pharmacy services conducted by HPs is the clinical pharmacist service (CPS). The CPS consists of a pharmacist compiling the medication history, medication reconciliation and medication review [28]. Pharmacist interventions following the service are documented in the patient's health record as a pharmacist note. Because collaboration between admission HPs and CPs does not yet exist in Region Zealand, it was deemed necessary to propose a way for the HPs and CPs to work together in relation to discharged patients and use this cooperation as an offset for discussion in the focus group interviews (see figure 1). 


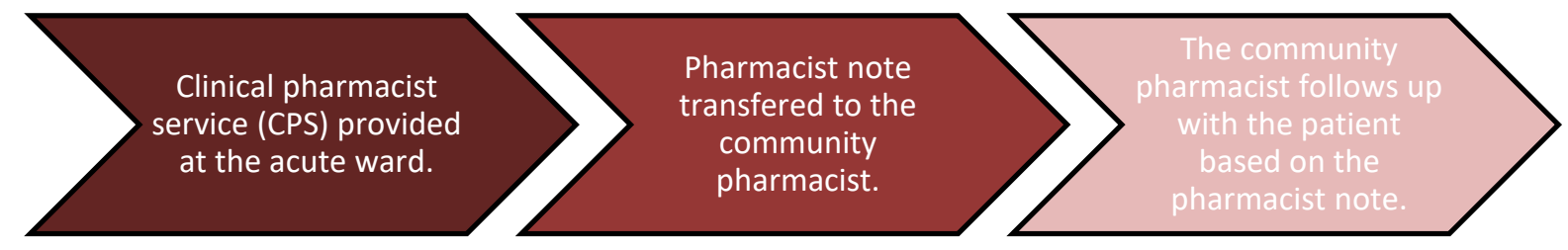

Figure 1: The proposed setup of an intraprofessional cross-sector collaboration between hospital and community pharmacists, as it was visually presented for the focus group participants. A pharmacist note is a note registered by a clinical pharmacist in the patient's electronic health record after conducting a medication review. It includes suggestions for interventions regarding medical treatment.

\section{Participants}

A purposeful sampling method was used, inviting HPs providing the CPS from the Region Zealand Hospital Pharmacy and CPS from all community pharmacies in the region [33]. Thirteen HPs and 38 CPs were invited to participate. Six HPs and nine CPs signed up to participate, allowing for two focus group interviews. Five HPs and six CPs were able to attend either of the two focus group interviews. The focus group interviews aimed at an equal distribution of HPs and CPs in each group [32]. LVJL facilitated both focus groups, while LSN moderated, took notes and observed the participants during the interview and made debriefing interviews with LVJL following each focus group interview.

\section{Data collection}

The focus groups were audio-recorded. Informed consent was signed by each participant before the groups were formed. A semi-structured interview guide based on an inductive approach was used (See Appendix I). The wording of the questions and the setup followed the focus group interview guide by Huston et al. [32]. Since the focus groups consisted of a mix of HPs and CPs, an "ice breaking" session was added at the beginning of the focus group (Section 2), allowing the participants to establish common ground (See Appendix I).

\section{Data analysis}

Audio recordings from both focus groups were transcribed verbatim. NVivo 10 (QSR International Pty Ltd, Australia) guided both the transcription and the analysis of the data. The data were analyzed using thematic analysis as suggested by Braun \& Clarke following their 6-phase guide for thematic analysis $[34,35]$. The thematic analysis was conducted deductively to identify data from existing collaboration theory in the literature $[36,37]$. Thus, emerging themes were arranged according to one of the six predefined theory-derived themes (see table 1). The derivation of the six themes from theory is described in the subsection "Theory" below. However, it was necessary to complement the analysis with an inductive thematic analysis, with more open coding, to accommodate themes that did not fit into the predefined theory-derived themes. The data were thus also searched for non-theory-derived factors affecting the collaboration.
LVJL and LSN first coded the transcript from the first focus group interview individually based on the predefined themes. Disagreements identified were discussed until consensus was reached. Then, LVJL and GRH individually coded passages of the transcript from the second focus group interview. Disagreements were discussed until a consensus was reached. Finally, LVJL, LSN and GRH met and discussed the emerging themes. Subsequently, LVJL discussed the final themes, subthemes and descriptions based on both focus group interviews with GRH and LSN to ensure confirmability of the analysis and subsequently the results [35].

\section{Theory}

LVIL conducted a literature search in PubMed and Google Scholar, which resulted in the identification of three collaboration theories on factors influencing collaboration. The theories had to be specific to either intra- or interprofessional collaboration and on collaboration in general within a team. Two non-healthcare-specific theories were identified $[37,38]$ alongside one healthcare-specific theory [36]. In the nonhealthcare-specific theory described by Mattessich et al., the authors identified no less than 19 different factors affecting collaboration, which was deemed too complex to apply for the analysis [38]. However, the factors that Mattessich et al. [38] described as affecting collaboration were comparable to many aspects with the other non-healthcare-specific theory by Patel et al. [37], which described eight factors. Thus, it was decided to use the non-healthcare-specific theory-derived factors described by Patel et al. [37] at the outset and to supplement and merge the theory with the factors described in the healthcare-specific theory by Morley et al. [36]. The merging of the two theories was deemed necessary for the resulting theory to fit the clinical and practice setting of HPs and CPs. The factors derived from the theories by Patel et al. [37] and Morley et al. [36] are listed in appendix II. The determinants from the theory described by Morley et al. were merged with the factors described in the theory by Patel et al. [37]. The merging resulted in six predefined themes used for the theoretic thematic analysis (see table 1). 
Table 1: The six predefined themes used for deductive analysis after merging of the factors described in the non-healthcare specific theory by Patel et al. [37] and the health-care specific theory by Morley et al. [36]:

$\mathrm{HP}=$ Hospital Pharmacist, $\mathrm{CP}=$ Community pharmacist

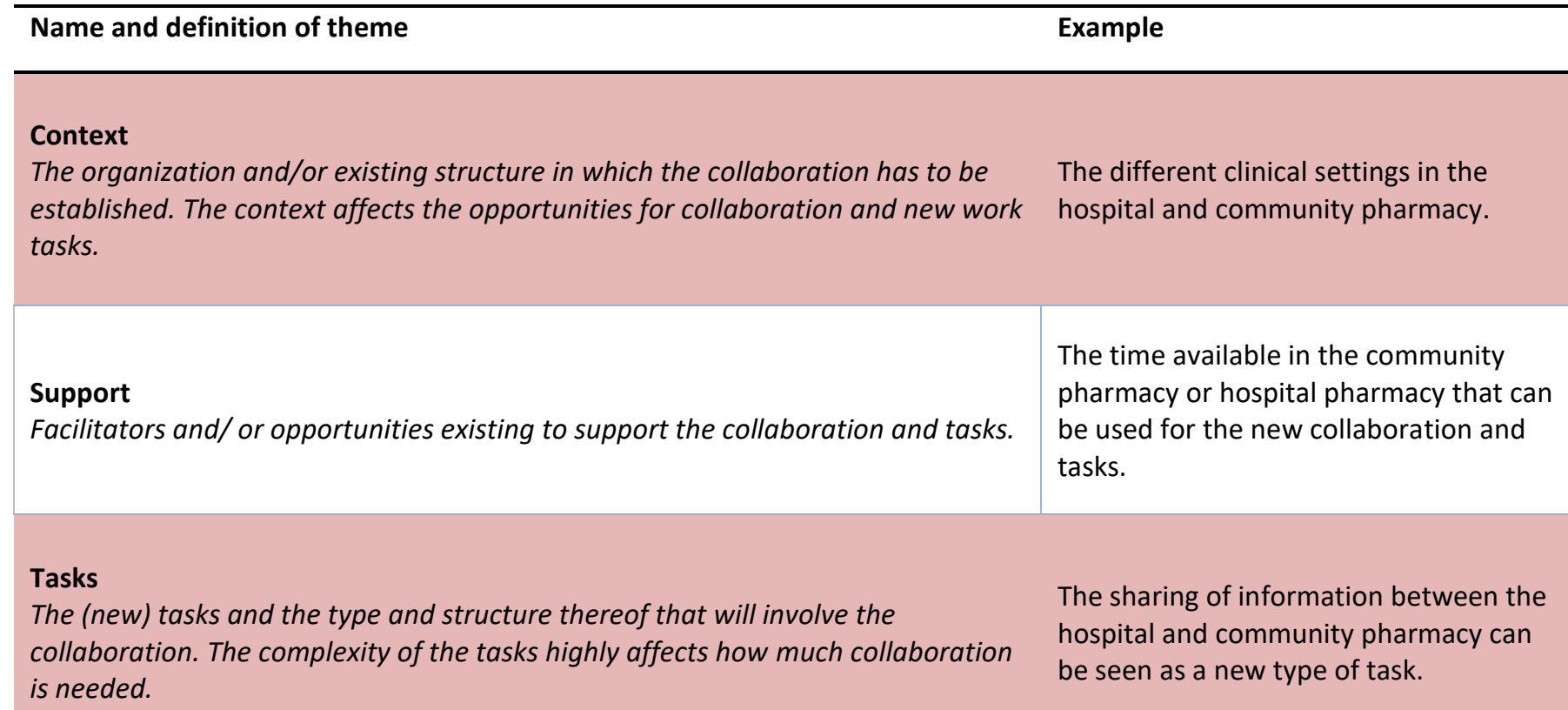

\section{Communication}

The type, frequency and need for communication in the collaboration. Successful communication makes collaboration more effective.

The frequency in which the HPs and CPs have to communicate for the collaborative tasks to be solved.

\section{Teams}

The nature and setup of the team that will collaborate, including the roles taken on by each team member and the understanding of each role in the team. Understanding the importance of and necessity of a role in a team affects the success of teamwork in the collaboration.

\section{Individuals}

The individual collaboration skills of the individuals in the team. Psychological factors such as motivation, beliefs and experience also affect the success of the

The motivation for collaborating with HPs/CPs. collaboration. 


\section{RESULTS}

Two focus group interviews were conducted in June 2017. They lasted 2 hours and $25 \mathrm{~min}$ and 2 hours and $43 \mathrm{~min}$. The participants were seven pharmacists (three HPs and four CPs) and four pharmacists (two HPs and two CPs). The pharmacists were on average 36 [30.6; 41.6] years old, the majority were females $(91.5 \%)$, and the HPs and CPs had an average of 5.2 [2.9; 7.6, respectively] years of experience in total.

Both groups of pharmacists were generally positive towards working intraprofessionally and expressed a willingness to collaborate across sector boundaries focusing on hospital- discharged patients. Both groups of pharmacists were interested in understanding the views and perspectives expressed by one another.

Three themes and seven subthemes (see figure 2) were identified. Two themes, "Individuals" and "Context" were theory-derived, while the theme "The hospital physicians and GPs as the focal points of the HPs' and CPs' work", was specific for this type of pharmacist collaboration and did not fit into preexisting themes. In the following section, the three themes and subthemes will be presented.

Figure 2: The three identified themes and subsequent subthemes.

Abbreviations: $\mathrm{CP}=$ Community Pharmacist, $\mathrm{HP}=$ Hospital Pharmacist, $\mathrm{GP}=$ General Practitioner

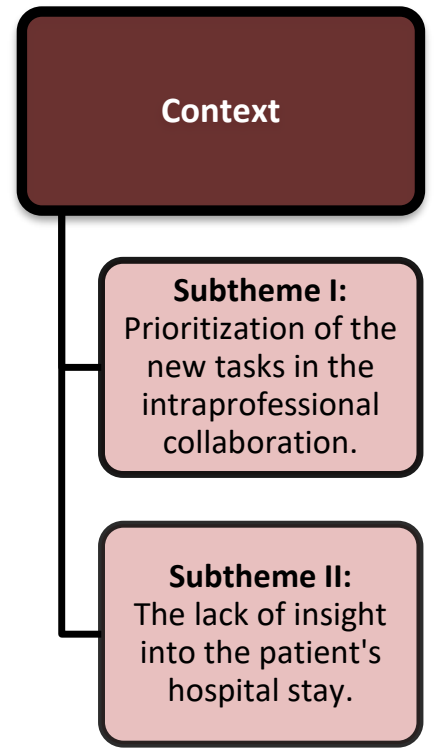

\section{Context}

The context in which the collaboration was to be established was important for both groups of pharmacists. They identified limitations in the way they already worked in the community pharmacy and on the hospital wards. It was important for both groups to gain insight into these limitations, as these limitations affected the possibilities for a new intraprofessional collaboration.

Prioritization of the new tasks in the intraprofessional collaboration

Both groups of pharmacists had limited time and resources to manage their current workload, and they were used to prioritize what information to provide to the hospital physician and/or the patient. Accordingly, this prioritization would need to be carried out in the intraprofessional collaboration as well.

The hospital physicians

and GPs as the focal

points of the HPs' and CPs' work

Subtheme I: The limitation of CPS and HPs are based on current roles and organizations.

Subtheme II: Lack of regular access to the GP gives the patients more responsibility.

Subtheme III: Lack of support from the GPs on the work conducted by HPS and CPs.

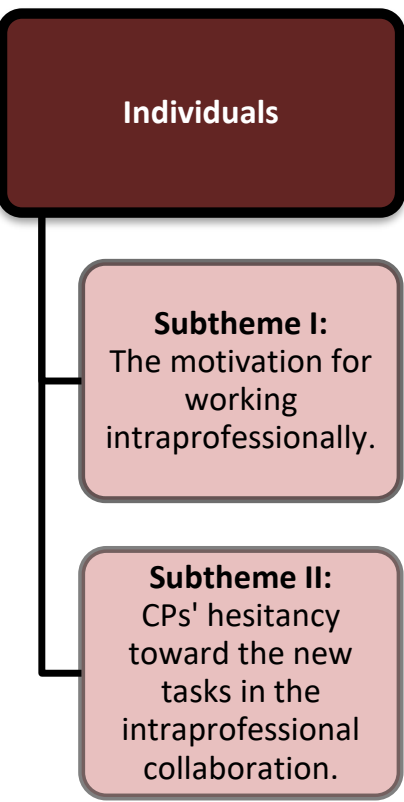

"(...) if I am to inform this poor customer about six different drugs at once (...) I mean, it is difficult to talk about all that in just one conversation because they are able to remember what $1 \%$ of what we tell them. "(CP-4)

Although both groups of pharmacists asked for a prioritization in relation to tasks in the intraprofessional collaboration, the HPs expected the prioritization to be different from current practice.

"Then, I just think about prioritizing because, when I prioritize, I do it with the hospital physician in mind. If I have to do that for a community pharmacist, then I would probably prioritize differently, I think." (HP-2) 
The lack of insight into the patient's hospital stay limits the HPs' and $\mathrm{CPs}^{\prime}$ abilities to give advice

The HPs providing the CPS at the acute ward lack insight into what happens to the patients and their medications after they leave the acute ward for the bed unit. Because of this lack of knowledge, the pharmacists were afraid of confusing the patients.

“(...) you don't know what the hospital physician implements and doesn't implement and then what? (...) Then, the community pharmacist at the community pharmacy has to convince the patient that something has to be changed in his/her medical treatment, even though a physician already decided nothing should be changed." (HP-4)

Insight into the work tasks of the other pharmacist group would make it easier for both groups to understand the possibilities for collaborating intraprofessionally and more easily build upon or supplement each other's work.

"I have only really worked in community pharmacy and sometimes, it is hard for me to understand what you, as hospital pharmacists, are really doing at the hospital (...) How can I at the community pharmacy also help get your interventions to the patients?" (CP-2)

\section{The hospital physicians and GPs as the focal points for the HPs' and CPs' work}

Both groups of pharmacists feel dependent on both the hospital physicians and GPs in their work because they need them to accept and implement their suggestions for changing the medication regimen. This dependency creates a strong focal point around the hospital physician and the GP in the work of both groups of pharmacists.

The limitations of CPs and HPs are based on current roles and organizations

Both groups of pharmacists acknowledged that the GPs and the hospital physicians are responsible for the medical treatment of the patient and are the only ones who can and should change or intervene in the medical treatment directly. Both groups of pharmacists see this dependency on the HPs and the GPs as limiting the success of an intraprofessional collaboration. If the hospital physicians and the GPs cannot be directly involved in the collaboration, the GPs should at least be informed about what happened to their patients at the community pharmacy.

"I think it is really good, this pharmacist-to-pharmacist collaboration. But I am just still afraid that these physicians are sitting in the middle really. How do we move forward, right? (...) We [the pharmacists] can't change the prescription (...) So I am just afraid about (...) that all these good intentions might just fall to the ground, because how do we get access to those physicians?" (HP-1)
Lack of regular access to the GP gives the patients more responsibility

Both groups of pharmacists have difficulty getting in contact with the GP. Therefore, an important aspect of intraprofessional collaboration should be the establishment of an easy way to get through to the GP with the medication changes proposed by either the HPs or the CPs. Both groups of pharmacists state that if it is impossible for them in the intraprofessional collaboration to get through to the GP, the patients should be responsible for communicating the suggestions for changes to the medical treatment made by HPs or CPs to the GP.

"I also think it might be important that we agree upon who is responsible for implementing this intervention, if we agree that you [the HPs] send something to us, that we should make an intervention on (...) do we tell the costumer: 'Be sure to talk with your GP about this'. Or do we directly contact [the GP]? (...) because otherwise, we will end up talking about something that could be a good idea, but then, it doesn't go any further than that." (CP-3)

Lack of support from the GPs for the work conducted by HPs and CPs

Both groups of pharmacists identified the lack of willingness from especially the GPs to collaborate. This lack of willingness is thought to occur because the GPs and the hospital physicians have a strong professional pride in their work and because the pharmacist profession has not branded itself enough.

“(...) and then we are stuck again with the fact that we haven't been good about communicating to others what we are doing and what we are capable of doing in relation to the physicians... so that they would understand that what we do is smart." (CP-3)

\section{Individuals}

The individual factors affecting the intraprofessional collaboration were related to motivation and hesitancy. The two groups of pharmacists were motivated differently for establishing an intraprofessional collaboration as a consequence of the difference in the way they are working. Additionally, the CPs felt hesitant about conducting new tasks in relation to the intraprofessional collaboration.

The motivation for working intraprofessionally

Due to the differences in their work tasks, both groups of pharmacists are motivated differently. The CPs are highly motivated by getting feedback from patients, so they know that they are making a difference for the individual patient. The HPs are not in the same way in contact with the individual patient and do not get their work directly validated by the hospital physician. However, natural trust from HPs towards CPs already exists, as it already does from HPs towards hospital physicians. 
“(...) we already write all this [to the hospital physician], where we hope that someone looks at it. And I am thinking that it would be in the same spirit, we would write to you [the CP] (...) of course, it would be nice to have feedback, but I would also think that I had the trust, the faith, that you [the community pharmacists] would do something good with it really." (HP-2)

CPs' hesitancy towards the new tasks in the intraprofessional collaboration

CPs felt hesitant about the new tasks related to the intraprofessional collaboration. This sentiment was especially evident when the HPs and the CPs discussed whether the new collaboration should lead to a new service or a consultation offered to hospital-discharged patients. To overcome this hesitancy, the CPs tried to relate to and make the new service look similar to an existing service.

"I am thinking it [the new service] could very much be like the New Medicines Service (...). Now, we just have some more information. (...) I mean it is positive, it is easier to fit into the community pharmacy." (CP-5)

\section{DISCUSSION}

In this study, we found that both HPs and CPs are generally positive towards intraprofessional collaboration at the interface between hospital and primary care. However, both groups of pharmacists question whether intraprofessional collaboration will actually change current practice. While intraprofessional collaboration may possibly solve some issues related to the transition of care, pharmacists acknowledge their dependency on physicians (either GPs or hospital physicians) to implement their work. This finding is highly relevant when developing the intraprofessional collaboration further, as this directly questions the implementation ability and feasibility of a solely intraprofessional collaboration between pharmacists.

The results show that a factor affecting the success and willingness to collaborate for the pharmacists was related to having insight into the work of the other pharmacist group and into the patient's hospital stay. Currently, the HPs in the Zealand region are only involved during the patients' admission process at the hospital. Meanwhile, CPs are only involved in dispensing medication postdischarge with no knowledge of what happened during the hospital stay. The need for more insight expressed by CPs is also reported in the literature, stating that community pharmacists lack sufficient information on hospital-discharged patients [39].

In other countries, HPs are increasingly involved in more than just fragments of the hospital stay and are a part of the interdisciplinary health care teams routinely conducting medication reviews at admission, during the hospital stay and at discharge $[40,41]$. A recent Danish study showed a significant effect on readmissions when the involvement of a pharmacist was multifaceted, i.e., when the HP was involved both during the hospital stay and at discharge [8]. In the Netherlands,
Ensing et al. reported a new intervention that also involved collaboration between HPs and CPs, enabling staff at the community pharmacy to gain insight into what happened with the patient's medication throughout the hospital stay. However, the referral and the collaboration were established between a pharmacist in the discharge process and the community pharmacist. The CPs conducted a follow-up with the patient, where they identified several drug-related problems post discharge. These drug-related problems were identified because the CPs were aware of medication changes and pending drug-related problems from the hospital stay information provided by the HPs $[24,42]$. In the present study, both HPs and CPs were concerned that the HPs do not have insight into what happened with the patient after admission. This concern combined with the above mentioned studies shows that a cross-sectorial intraprofessional collaboration could be made more relevant in relation to enhancing medication safety for discharged patients if the collaboration could be established between a HP in the discharge process and a $\mathrm{CP}$ at the community pharmacy.

In this study, the hospital physicians and the GPs seemed to be the focal points of the pharmacists' work, which is right now limiting both groups of pharmacists' tasks even in a solely intraprofessional collaboration. Missing access to and support from the GPs in general suggest that the interprofessional collaboration still has to be optimized, especially in relation to the CPs. Based on these results, one might hypothesize that a fruitful intraprofessional collaboration comes from a wellestablished interprofessional collaboration. Nørgaard et al. suggested that for the community pharmacy to expand their role, CPs would have to focus more on interprofessional collaboration with GPs [43]. However, Gobis et al. also argued that too much effort has been put into widening interprofessional collaboration while HPs and CPs are taking very divergent paths, almost becoming two different professions due to less or too little focus on intraprofessional collaboration [21]. The divergent paths taken by the HPs and the CPs are also described by Taylor et al., who claim that HPs are becoming increasingly specialized [44]. According to Gobis et al., this specialization results in a siloed and fragmented approach to patient care opposed to seamless, coordinated and continuous patient care, which is advocated to reach optimal patient care [44]. The divergent paths taken by HPs and CPs might also be why HPs and CPs lack knowledge about each other's work, as our findings suggest.

Important measures may be implemented to create a fruitful offset for intraprofessional collaboration. It is necessary to introduce intraprofessional pharmacist collaboration during pharmacy education. The WHO has stressed the need for interprofessional education, also known as IPE [45], and this has been implemented in pharmacy education in some countries (e.g., USA and UK), where pharmacists, nurses and physicians work with clinical cases to enhance their interprofessional skills [46]. Emphasizing intraprofessional collaboration during the 
pharmacist curriculum is also vital, as this would enable pharmacists to seek collaboration with pharmacists in other settings after graduation, and they would have more opportunities to address challenges regarding patients intraprofessionally. After graduation, it is important for the profession to foster awareness of the different work tasks conducted by pharmacists in various settings and specialties. This could be facilitated by allowing pharmacists to shadow each other's work and to create networks with the aim of sharing knowledge between HPs and CPs. The new insight may provide CPs and HPs with new ideas for collaboration to enhance medication safety at care transitions.

In the present study, we found that the HPs an CPs lacked knowledge about each other's clinical services and competencies. Furthermore, a lack of support from other healthcare professionals (i.e., physicians) detracted intraprofessional collaboration. This was also shown in a survey by Penm et al. [47], where pharmacists were highly affected by the views of other professions in relation to expansion of their role. It is surprising to see in our study that even when focusing on intraprofessional collaboration, CPs and HPs are intentionally or unintentionally affected by physicians in patient care. Thus, our study also indicates that to create an environment conducive to intraprofessional collaboration among pharmacists, it is necessary to change the views of pharmacists themselves regarding their own competencies and abilities.

\section{Strengths and limitations}

In the present study, both the facilitator and the moderator (LVIL and LSN) were trained pharmacists and thus had insight and knowledge about the pharmacy field, strengthening the study results. To overcome the possibility that too much insider knowledge might be brought into the analysis of data, another health care professional (GRH) was involved in the analysis, securing confirmability in the analysis phase [32]. Additionally, preconceptions were discussed prior to the first focus group between the moderator and facilitator, to make sure these were unintentionally taken into the focus groups and affected the results. After each focus group, the moderator and facilitator conducted a debriefing session. After the first focus group, better probes to induce discussion were added to the interview guide. Additionally, the introduction and information about the study was shortened to make space for more fruitful discussions.

In the present study, the derived themes from existing collaboration theory fit our study results to only a minor extent. Only two out of six of the theory-derived themes were identified in our data namely "Context" and "Individuals". "Context" seemed to be an important theme affecting the establishment of intraprofessional collaboration, and it might be that the HPs and CPs had to comprehend the context and the differences in context prior to being able to discuss other themes, such as tasks, communication and support. However, the deductive analysis was supplemented with an inductive analysis, allowing for new themes not based on theory to arise. This process highly strengthened the output of the analysis and subsequently the credibility of the results.

The study also had limitations. We invited only HPs employed in one hospital pharmacy in one of the five regions in Denmark, which limited the number of HP informants available. Consequently, we ran out of HP informants for more focus groups but had several CP participants available. This limitation, together with the number of focus groups held, might affect the credibility of the study results, as the number of focus group interviews conducted was a consequence of the available pool of informants and not a matter of reaching data saturation [35].

Future studies should preferably aim at conducting more focus groups with the inclusion of HPs and CPs from different geographical regions to enhance saturation and transferability of the study results [34]. Additionally, it is relevant to study whether successful and well-implemented interprofessional collaboration between physicians, HPs and CPs can enable better intraprofessional collaboration. Lastly, piloting a collaboration between HPs and CPs and exploring HPs' and CPs' views on a specific collaboration in real world practice is recommended.

\section{CONCLUSION}

Hospital pharmacists (HPs) and community pharmacists (CPs) are highly influenced by the context in which their work is embedded in establishing a new intraprofessional collaboration. Additionally, they miss insight into each other's work in order to be able to see the full potential of working intraprofessionally. While HPs and CPs are in general positive towards intraprofessional collaboration, physicians are important key professionals that both HPs and CPs depend on when they conduct their work. This dependency of physicians seems to affect the pharmacists' views on the capability and success of a solely intraprofessional collaboration. Future studies should aim at piloting a specific collaboration between HPs and CPs and explore their views and perspectives toward collaborating in real world practice.

Treatment of Human Subjects: IRB review/approval required and obtained

Conflicts Of Interest: We declare no conflicts of interest or financial interests that the authors or members of their immediate families have in any product or service discussed in the manuscript, including grants (pending or received), employment, gifts, stock holdings or options, honoraria, consultancies, expert testimony, patents and royalties. 


\section{References}

1. Bullock S, Morecroft CW, Mullen R, Ewing AB. Hospital patient discharge process: an evaluation. Eur J Hosp Pharm Sci Pract. 2017 Sep;24(5):278-82.

2. Bruhwiler LD, Hersberger KE, Lutters M. Hospital discharge: What are the problems, information needs and objectives of community pharmacists? A mixed method approach. Pharm Pract (Granada). 2017 Sep;15(3):1046.

3. Michel B, Hemery M, Rybarczyk-Vigouret M-C, Wehrlé $P$, Beck $M$. Drug-dispensing problems community pharmacists face when patients are discharged from hospitals: a study about 537 prescriptions in Alsace. Int J Qual Health Care. 2016 Dec 1;28(6):779-84.

4. Ziaeian B, Araujo KLB, Van Ness PH, Horwitz LI. Medication reconciliation accuracy and patient understanding of intended medication changes on hospital discharge. J Gen Intern Med. 2012 Nov;27(11):1513-20.

5. Maxwell K, Harrison J, Scahill S, Braund R. Identifying drug-related problems during transition between secondary and primary care in New Zealand. Int J Pharm Pract. 2013 Oct;21(5):333-6.

6. Ahmad A, Mast MR, Nijpels G, Elders PJ, Dekker JM, Hugtenburg JG. Identification of drug-related problems of elderly patients discharged from hospital. Patient Prefer Adherence. 2014;8:155-65.

7. MacDonald K, Cusack M, Liang SQR, Rinco K. Care Gaps in the Electronic Discharge Medication Reconciliation Process at an Acute Care Facility. Can J Hosp Pharm. 2017 Dec;70(6):430-4.

8. Ravn-Nielsen LV, Duckert M-L, Lund ML, Henriksen JP, Nielsen ML, Eriksen CS, et al. Effect of an In-Hospital Multifaceted Clinical Pharmacist Intervention on the Risk of Readmission: A Randomized Clinical Trial. JAMA Intern Med. 2018;178:375-82.

9. Cavanaugh JJ, Lindsey KN, Shilliday BB, Ratner SP. Pharmacist-coordinated multidisciplinary hospital follow-up visits improve patient outcomes. J Manag Care Spec Pharm. 2015 Mar;21(3):256-60.

10. Nazareth I, Burton A, Shulman S, Smith P, Haines A, Timberal H. A pharmacy discharge plan for hospitalized elderly patients--a randomized controlled trial. Age Ageing. 2001 Jan;30(1):33-40.

11. Neeman $M$, Dobrinas $M$, Maurer $S$, Tagan $D$, Sautebin A, Blanc A-L, et al. Transition of care: A set of pharmaceutical interventions improves hospital discharge prescriptions from an internal medicine ward. Eur J Intern Med. 2017 Mar;38:30-7.

12. Phatak A, Prusi R, Ward B, Hansen LO, Williams MV, Vetter $E$, et al. Impact of pharmacist involvement in the transitional care of high-risk patients through medication reconciliation, medication education, and postdischarge call-backs (IPITCH Study). J Hosp Med. 2016 Jan;11(1):39-44.
13. Ensing $H T$, Stuijt $C C M$, van den Bemt BJF, van Dooren $A A$, Karapinar-Carkit $F$, Koster ES, et al. Identifying the Optimal Role for Pharmacists in Care Transitions: A Systematic Review. J Manag Care Spec Pharm. 2015 Aug;21(8):614-36.

14. Melody KT, McCartney E, Sen S, Duenas G. Optimizing care transitions: the role of the community pharmacist. Integr Pharm Res Pract. 2016;5:43-51.

15. American College of Clinical Pharmacy, Hume AL, Kirwin J, Bieber HL, Couchenour RL, Hall DL, et al. Improving care transitions: current practice and future opportunities for pharmacists. Pharmacotherapy. 2012 Nov;32(11):e326-337.

16. Kristeller J. Transition of care: pharmacist help needed. Hosp Pharm. 2014 Mar;49(3):215-6.

17. Supper I, Catala O, Lustman M, Chemla C, Bourgueil Y, Letrilliart L. Interprofessional collaboration in primary health care: a review of facilitators and barriers perceived by involved actors. J Public Health (Oxf). 2015 Dec;37(4):716-27.

18. Hwang AY, Gums TH, Gums JG. The benefits of physician-pharmacist collaboration. J Fam Pract. 2017 Dec;66(12):E1-8.

19. Christensen M, Lundh A. Medication review in hospitalised patients to reduce morbidity and mortality. Cochrane Database of Systematic Reviews [Internet]. 2016;(2). Available from: https://doi.org//10.1002/14651858.CD008986.pub3

20. Graabaek T, Kjeldsen LJ. Medication reviews by clinical pharmacists at hospitals lead to improved patient outcomes: a systematic review. Basic Clin Pharmacol Toxicol. 2013;112:359-73.

21. Gobis B, Yu A, Reardon J, Nystrom M, Grindrod K, McCarthy L. Prioritizing intraprofessional collaboration for optimal patient care: A call to action. Can Pharm J (Ott). 2018 Apr 2;151(3):170-5.

22. Judith Kristeller, Felicia Snyder, Fanhui Kong, Michele Musheno. Collaboration between Hospital and Community Pharmacists to Improve Medication Management from Hospital to Home. INNOVATIONS in Pharmacy. 2017;8(2).

23. Ensing $H T$, Koster ES, Dubero DJ, van Dooren AA, Bouvy ML. Collaboration between hospital and community pharmacists to address drug-related problems: The HomeCoMe-program. Res Social Adm Pharm. 2018 May 8;

24. Ensing HT, Koster ES, Stuijt CCM, van Dooren AA, Bouvy ML. Bridging the gap between hospital and primary care: the pharmacist home visit. Int J Clin Pharm. 2015 Jun;37(3):430-4.

25. Nazar H, Brice S, Akhter N, Kasim A, Gunning A, Slight $\mathrm{SP}$, et al. New transfer of care initiative of electronic referral from hospital to community pharmacy in England: a formative service evaluation. BMJ Open. 2016 14;6(10):e012532. 
26. Booij AD, de Boer WO, Kokenberg MEAP, Tromp TFJ. Interventions in seamless care. Pharm World Sci. 2003 Apr;25(2):41-2.

27. Frontini R, Miharija-Gala T, Sykora J. EAHP survey 2010 on hospital pharmacy in Europe: parts 4 and 5. Clinical services and patient safety. European Journal of Hospital Pharmacy: Science and Practice. $2013 \mathrm{Apr}$ 1;20(2):69-73.

28. Nielsen TRH, Andersen SE, Rasmussen M, Honore PH. Clinical pharmacist service in the acute ward. Int J Clin Pharm. 2013 Dec;35(6):1137-51.

29. Smith F. Health Services Research Methods in Pharmacy: Focus groups and observation studies. International Journal of Pharmacy Practice. 2011 Feb 22;6(4):229-42.

30. Catherine Pope NM. Foucs grouos. In: Qualitative research in health care. 3rd ed. United Kingdom: Blackwell Publishing; 2006. p. 156.

31. Pharmacies [Internet]. Danish Medicines Agency. [cited 2019 Jul 12]. Available from: https://laegemiddelstyrelsen.dk/en/pharmacies/phar macies/

32. Boyd M, Waring J, Barber N, Mehta R, Chuter A, Avery AJ, et al. Protocol for the New Medicine Service Study: a randomized controlled trial and economic evaluation with qualitative appraisal comparing the effectiveness and cost effectiveness of the New Medicine Service in community pharmacies in England. Trials. 2013 Dec 1;14:411.

33. Malterud K. Qualitative research: standards, challenges, and guidelines. Lancet. 2001 Aug 11;358(9280):483-8.

34. Braun V, Clarke V. Using thematic analysis in psychology. Qualitative Research in Psychology. 2006 Jan 1;3(2):77-101.

35. Guba EG. Criteria for assessing the trustworthiness of naturalistic inquiries. ECTJ. 1981 Jun 1;29(2):75.

36. Morley L, Cashell A. Collaboration in Health Care. J Med Imaging Radiat Sci. 2017 Jun;48(2):207-16.

37. Patel H, Pettitt M, Wilson JR. Factors of collaborative working: a framework for a collaboration model. Appl Ergon. 2012 Jan;43(1):1-26.

38. Mattessich PW, Monsey BR. Collaboration: What Makes It Work. A Review of Research Literature on Factors Influencing Successful Collaboration [Internet]. St. Paul, Minnesota: Amberst H. Wilder Foundation; 1992 p. 57. Available from: https://eric.ed.gov/?id=ED390758
39. Brühwiler LD, Hersberger KE, Lutters M. Hospital discharge: What are the problems, information needs and objectives of community pharmacists? A mixed method approach. Pharm Pract (Granada). 2017 Sep;15(3):1046.

40. Bergkvist Christensen A, Holmbjer L, Midlöv $P$, Höglund $P$, Larsson $L$, Bondesson $\AA$, et al. The process of identifying, solving and preventing drug related problems in the LIMM-study. Int J Clin Pharm. 2011 Dec;33(6):1010-8.

41. Bergkvist $A$, Midlöv $P$, Höglund $P$, Larsson $L$, Bondesson A, Eriksson T. Improved quality in the hospital discharge summary reduces medication errors--LIMM: Landskrona Integrated Medicines Management. Eur J Clin Pharmacol. 2009 Oct;65(10):1037-46.

42. Ensing HT, Koster ES, Sontoredjo TAA, van Dooren AA, Bouvy ML. Pharmacists' barriers and facilitators on implementing a post-discharge home visit. Res Social Adm Pharm. 2017 Aug;13(4):811-819.e2.

43. Josefine D. Nørgaard, Sofia K. Sporrong. Views on the role of community pharmacy in local communities: a case study of stakeholders' attitudes. Pharm Pract (Granada) [Internet]. 2019 Jun 1 [cited 2019 Jul 10];17(2). Available from:

https://www.pharmacypractice.org/journal/index.ph $\mathrm{p} / \mathrm{pp} /$ article/view/1419

44. Spehar AM, Campbell RR, Cherrie C, Palacios P, Scott D, Baker JL, et al. Seamless Care: Safe Patient Transitions from Hospital to Home. In: Henriksen K, Battles JB, Marks ES, Lewin DI, editors. Advances in Patient Safety: From Research to Implementation (Volume 1: Research Findings). Rockville (MD): Agency for Healthcare Research and Quality (US); 2005.

45. World Health Organization. Framework for Action on Interprofessional Education \& Collaborative Practice [Internet]. Geneva: World Health Organization; 2010 p. 64. Available from: http://whqlibdoc.who.int/hq/2010/WHO_HRH_HPN_ 10.3_eng.pdf.

46. Patel N, Begum S, Kayyali R. Interprofessional Education (IPE) and Pharmacy in the UK. A Study on IPE Activities across Different Schools of Pharmacy. Pharmacy (Basel). 2016 Sep 26;4(4):28.

47. Penm J, Jorgenson D, MacKinnon NJ, Smith J. Part 1: Barriers to the advancement of the pharmacy profession. Can Pharm J (Ott). 2017 Apr 7;150(3):150-2. 


\section{Appendix I}

Interview guide used in the focus group interviews.

Subject

Aim(s)
Probe(s)

Method
1. Problem and solution

identification

2. The collaboration framework
To understand the views of the participants on the new collaborative tasks and the collaboration itself.
3. The aim and tasks of the collaboration
To make the pharmacists identify which problems and solutions the participants consider there are in relation to the transition of care and to assess whether they agree on the problems and solutions.
- Were there any problems presented by the other participants that you were not aware of, and if so, what are they?
- What is the main content of the best collaboration?

- What are the strengths and weaknesses of the collaboration?

- What are the possibilities and threats?

- Is it possible to solve some of the problems related to the transition of care through collaboration? Why/why not?
To understand what facilitators and barriers that arises from the collaboration framework as seen by HPs and CPs.
- What are the facilitators and barriers for the collaboration to succeed?

- Which conditions have to be met for the collaboration to be established?

- What should be the aim of the collaboration?

- Are there any unclear areas or any areas we have not touched upon?
The participants individually wrote down for 5-7 minutes what they considered the three major problems and solutions on paper.

This was followed by a plenary discussion.

Plenary discussion. The facilitator briefly described the collaboration model and an example of a pharmacist note.

Plenary discussion. A discussion prompted by examples of themes on the table they could discuss.

Plenary discussion.

\section{importance to the participants} were discussed. 


\section{APPENDIX II}

Factors described affecting collaboration based on the non-healthcare-specific theory by Patel et al.

(blue) [37] and the health-care-specific theory by Morley et al. (red) [36].
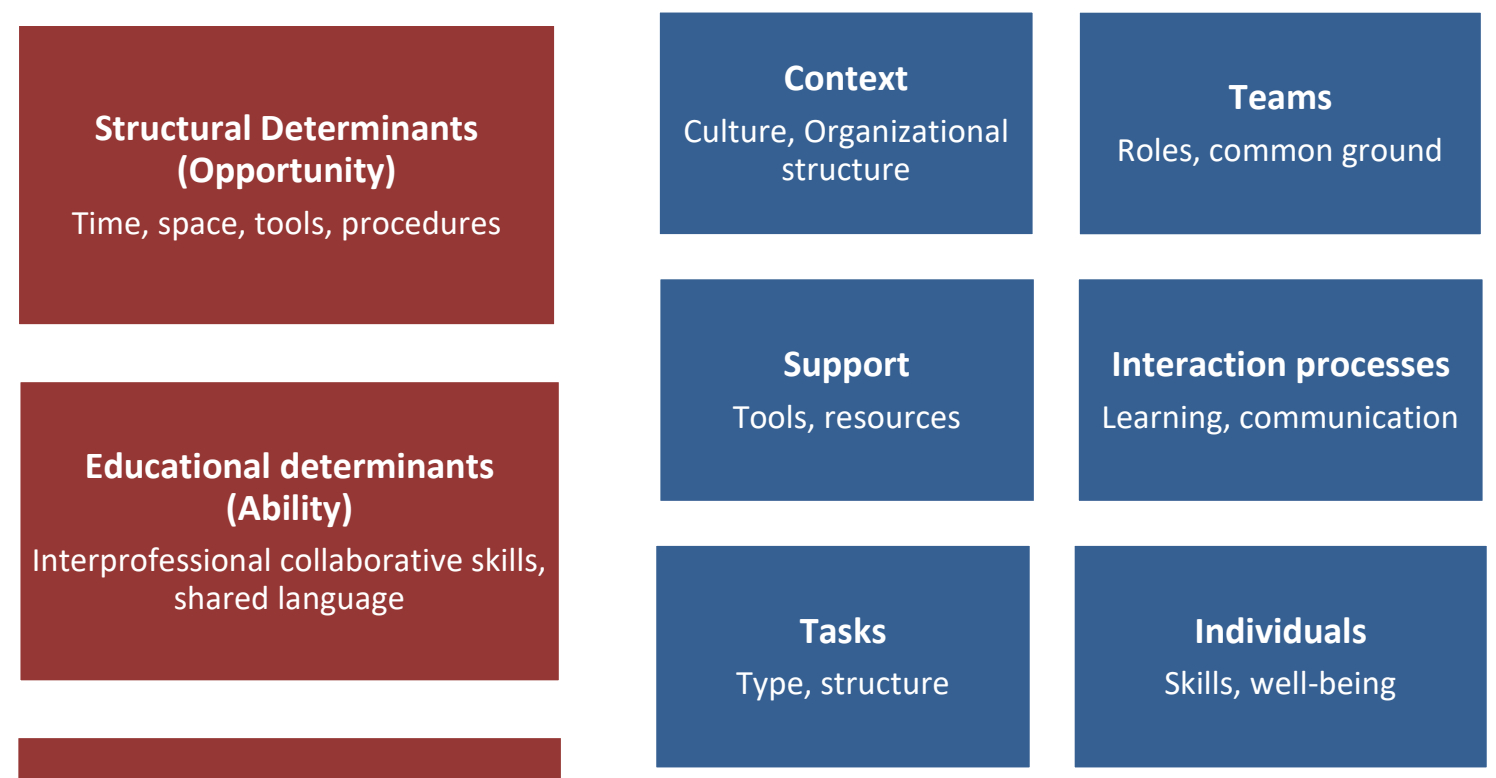

Physiological determinants (Willingness)

Safety, collegiality, role valuing

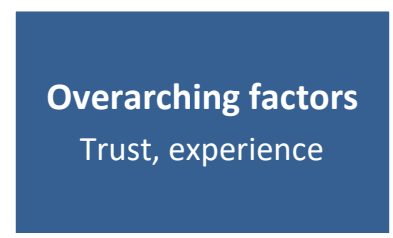

Patel et al (blue) suggests eight factors affecting the success of collaboration, each divided into different subfactors. Two examples of subfactors per factor are presented in this figure. In the original article, all the subfactors for each factor are described [37].

Morley et al. (red) suggest three determinants affecting the success of inter-professional collaboration, each supported by different specific determinants [36]. 